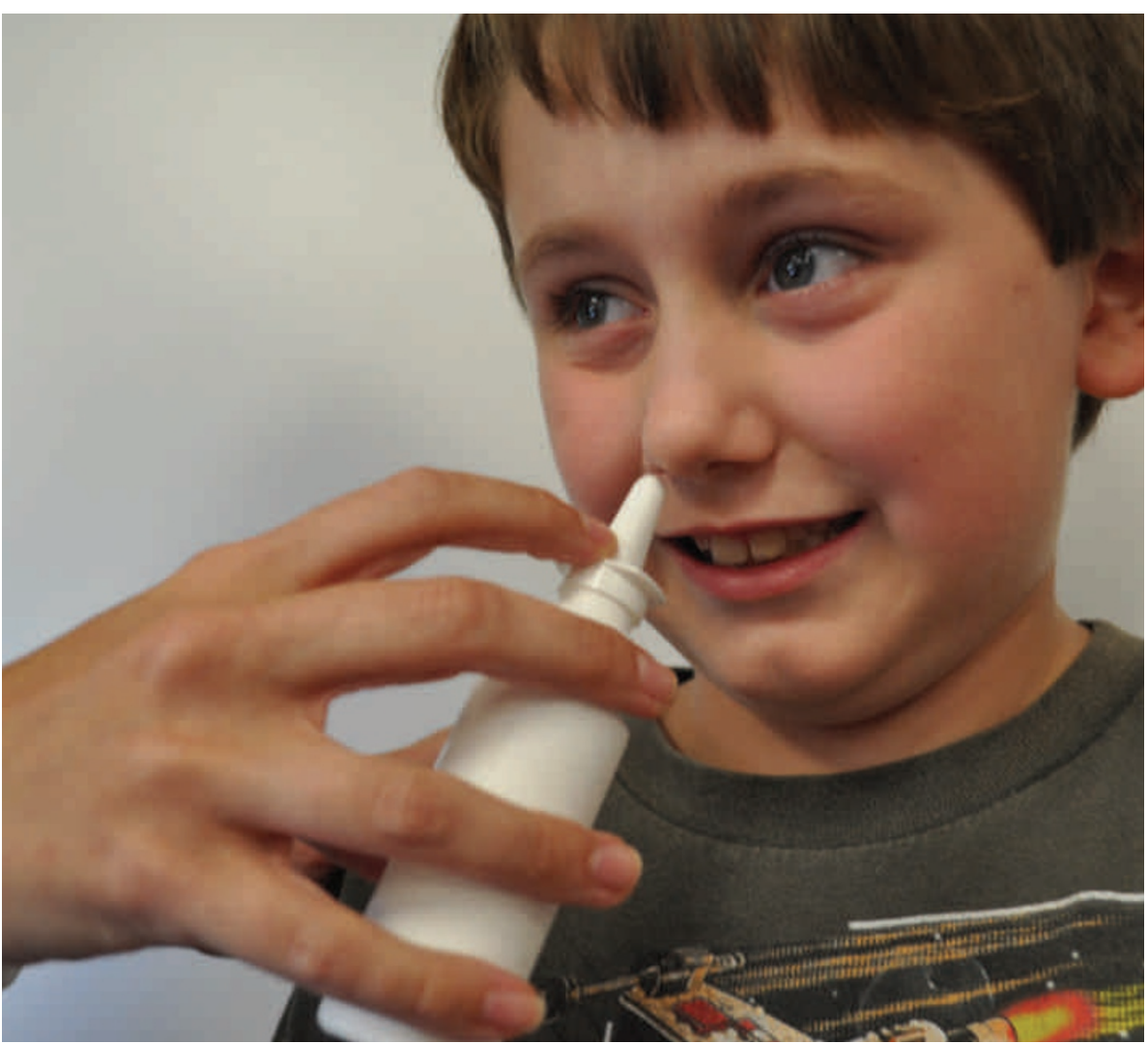

A seven-year-old boy with autism is given oxytocin nasally to see if it alters his sociability.

TREATMENTS

\title{
In the waiting
} room

\section{After years of making do with drugs developed for other conditions, doctors and scientists are eagerly pursuing drugs that target the social symptoms of autism.}

\section{BY MICHAEL EISENSTEIN}

$\mathrm{W}$ hy would any parent feed their child worm eggs? In Stewart Johnson's case, it was because he believed he had stumbled across a promising treatment for the symptoms of his teenage son's autism after limited success with other treatments. Although he is not a professional scientist, his bold experiment with porcine whipworm (Trichuris suis) ova has culminated in a formal clinical trial spearheaded by a leading autism research centre. However, Johnson's story also tells of the desperation felt by many parents to find something - anything — that might improve the lives of children with autism spectrum disorders (ASDs).
That desperation is borne of the limited success achieved in developing drugs to treat autism. "When I give a lecture on medication, I usually start by saying that I won't be talking about the state of pharmacology in autism, but about the miserable state of pharmacology in autism," says Antonio Hardan, a child psychiatrist at Stanford University School of Medicine in California. In fact, no drugs are approved by the US Food and Drug Administration (FDA) for autism per se, although a handful of medi-

\section{"Talling about immune stuff was considered fringe and funliy."}

have become good at stealing drugs developed for other disorders," jokes Hardan. But even second-hand drugs are better than nothing, given how disruptive these behaviours can be.

\section{STRIKING ABALANCE}

'Irritability' may sound like a minor failing, but the behaviours it encompasses - mood swings, aggression and self-harming - can be debilitating and dangerous to people with autism and their caregivers. That's why irritability is a primary target for pharmaceuticals in ASDs. Two antipsychotic drugs, aripiprazole (marketed as Abilify by Otsuka Pharmaceuticals) and risperidone are now in widespread use in people with ASDs. "These drugs are effective in $60-70 \%$ of children and adolescents that have significant irritability," says Christopher J. McDougle, director of the Lurie Center for Autism at Massachusetts General Hospital in Boston. Both drugs, however, come with side effects. Jeremy Veenstra-VanderWeele, a child psychiatrist and researcher at Vanderbilt University Medical Center in Nashville, Tennessee, has determined that aripiprazole and risperidone frequently induce drowsiness and weight gain, and he cautions against prescribing these drugs unless symptoms are severe. "When you're using a medicine in a seven-year-old child that increases their body weight by $10 \%$ over the course of a couple of months, that potentially changes the way their metabolism is going to work down the line," he says, noting the chance for increased risk of metabolic syndrome or cardiovascular disease.

Many clinicians prescribe anti-depressants from the selective-serotonin reuptake inhibitor (SSRI) family, such as fluoxetine (Prozac) and citalopram, to treat ASD patients, despite scant evidence supporting their use. Randomized controlled trials have shown that SSRIs may reduce compulsive, repetitive behaviour in adults with autism, but have no observed effect on these symptoms in children and adolescents. But these trials did not examine social anxiety, a symptom for which some doctors have observed improvements in children with SSRIs. McDougle, who pioneered the study of SSRIs for ASDs while working at Yale University School of Medicine in the 1990s, believes factors related to ageassociated brain development may also limit their use. "One reason some children didn't get better was that they couldn't tolerate even low starting doses," he says. "They became much more irritable, agitated and aggressive."

Since the value of both SSRIs and antipsychotics is based largely on the extent to which they enable children with autism to participate in school, caregivers must weigh the potential medical benefits and adverse effects carefully. "If we're putting kids to sleep, even if their aggression is down, we haven't actually facilitated their psycho-educational intervention," says Evdokia Anagnostou, a child 


\section{ACTIVATING THE AUTISTIC BRAIN}

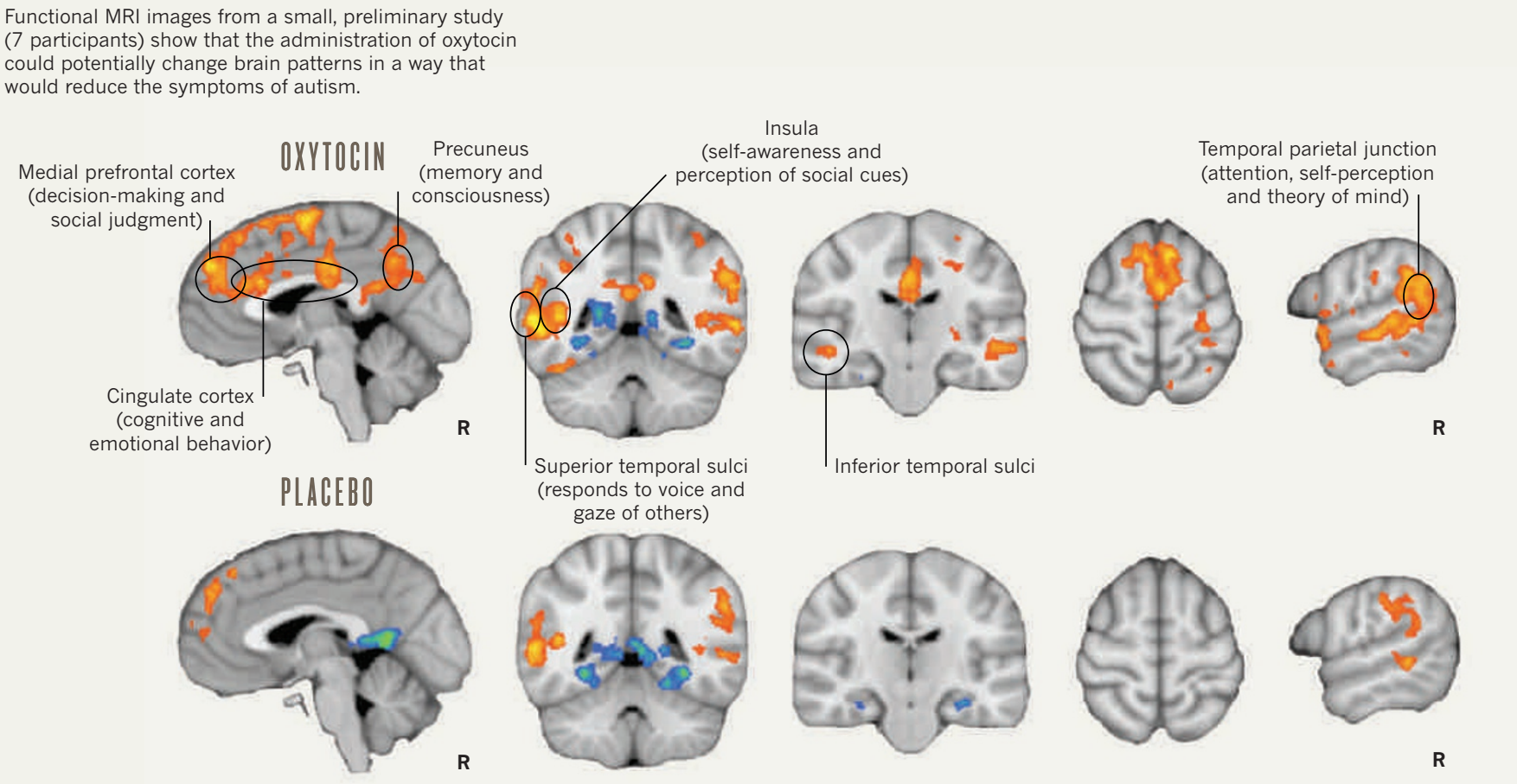

neurologist at the Bloorview Research Institute in Toronto, Canada.

\section{THE HEART OF THE MATTER}

No medications target the core symptoms of autism, which include attention deficit, abnormal social behaviour and poor ability to communicate. Accordingly, when a potential treatment option for these symptoms is identified, it grabs the community's attention. For example, extensive research in both animals and humans has identified a prominent role for the hormone oxytocin in behaviours associated with trust, empathy and attachment emotions commonly impaired in people with ASDs. At least half-a-dozen clinical trials are underway to test whether oxytocin affects these traits in people with ASDs.

The Swiss pharmaceutical company Novartis makes a nasal oxytocin spray, Syntocinon, and this is the primary mode of delivery in development. Veenstra-VanderWeele cautions that the benefits remain unclear. "Kids with ASD are having oxytocin squirted up their noses, and we need to know if that makes any sense," he says. Early studies have shown modest improvements - for example, an increased tendency to make eye contact or elevated activity in social centres of the brain. But most of these investigations have looked at the impact of single doses in small numbers of patients. A new round of larger-scale initiatives should help clarify both the efficacy and the longterm safety of oxytocin use. For example, a treatment network funded by the US National Institutes of Health through its Autism Centers of Excellence programme is set to embark on an ambitious five-year study of 300 children and adolescents to examine whether extended treatment with oxytocin confers measurable improvements in social function.

One of the biggest obstacles confronting clinical studies of oxytocin - and, indeed, any treatment of ASD's core symptoms - is the lack of robust, reproducible measurements of social deficits and improvements in those deficits. "Social behaviour is so complicated, and trying to measure changes in it is really tricky," says McDougle. This is especially true for early-stage experimental drugs, which target neurological pathways with unclear contributions to ASD and in which the therapeutic effects are likely to be weaker than for approved drugs such as risperidone. Furthermore, the inclusivist definition of autism as a spectrum of disorders overlooks very real differences. "Autism is a heterogeneous disorder, and it's very unlikely that you're going to find a compound that's effective in the treatment of social deficits in all kids with autism," says Hardan.

\section{FRAGILE X MARKS THE SPOT}

Some of the most exciting progress has emerged from work focused on ASDs with a clearly defined genetic cause. For example, research by Mark Bear, a neuroscientist at the Massachusetts Institute of Technology in Cambridge, has shown that defects in brain signalling mediated by the neurotransmitter glutamate appear to be critical to the pathophysiology of fragile X syndrome (FXS), an inherited disability in which patients often manifest autistic-like social deficits.
FXS is caused by mutations in the gene encoding a protein that regulates the production of other proteins involved in neuron signalling. Bear and colleagues discovered that the protein produced by the mutated gene represses a glutamate receptor called mGluR5. Bear hypothesized that the neurological symptoms of FXS might arise from overactive mGluR5, so his group studied animal models of FXS to test this theory". "We took fragile $\mathrm{X}$ mice and engineered them to have $50 \%$ of the normal level of mGluR5, with the prediction that this would correct multiple aspects of the disease, and it did," says Bear. "That was a powerful proof of principle."

Since then, numerous drug candidates have been identified that can potentially correct mGluR5-associated deficits - Novartis, Swiss drug giant Roche, and Seaside Therapeutics, based in Cambridge, Massachusetts, and co-founded by Bear, all have agents in clinical trials. Mouse models of FXS have proven useful in testing these candidates, as they directly mimic the genetic and cellular-scale defects seen in FXS, even if they do not always recapitulate the full spectrum of behavioural and social symptoms. Most encouragingly, a collaboration between Bear's team and Lothar Lindemann's research group at Roche has discovered that mGluR5 inhibitors can even repair FXS-associated symptoms in grown mice ${ }^{2}$ - suggesting that the window for repairing cognitive damage may be far wider than expected. "That's something we wouldn't have imagined 10 years ago," says Veenstra-VanderWeele, who was not involved in the study. 
Novartis has published data from a phase II trial of its mGluR5 inhibitor, AFQ056. The results suggest that the drug may have positive behavioural effects in a subset of adult FXS patients ${ }^{3}$, but the size of the cohort that benefited was too small to be conclusive. The company is now recruiting for a larger phase III trial. Seaside has also announced (but not published) encouraging phase II results in treating social symptoms of FXS with arbaclofen, a drug that inhibits glutamate release.

This spate of trials announces the entry of the pharmaceutical industry into a sector that it had long shied away from, owing to issues of complexity and a lack of concrete targets. "I think they are getting more interested - it's a huge market out there, and the science is getting intriguing," says neuroscientist Gerald Fischbach, scientific director of the New York-based Simons Foundation Autism Research Initiative. There is also hope, but not yet supporting evidence, that the deficits seen in FXS may share common roots with some other ASDs. "We need to understand the pathophysiology of each of these models and look for points of convergence, but we should not make assumptions about what those might be," says VeenstraVanderWeele. Nevertheless, the key role of glutamate signalling in the brain has given some scientists cause for optimism, and several trials are underway to examine the potential benefits of repurposing other known glutamatergic modulators. For example, Anagnostou and colleagues are conducting pilot studies with memantine (marketed as Namenda by Forest Laboratories) and riluzole (marketed as Rilutek by Sanofi-Aventis), which were approved by the FDA for Alzheimer's disease and amyotrophic lateral sclerosis respectively. "This is a very rich area to look for molecular targets," she says.

\section{PICKING UP HINTS}

All the same, there is a very real risk that treatments for patients with ASD subsyndromes such as FXS or the neurodevelopmental disorder Rett syndrome will benefit only a small subset of people with autism outside these categories. Some researchers further caution against drawing overly broad connections between these conditions that may be relatively superficial or even misleading. "Everybody says people with those syndromes also exhibit characteristics of autism," says McDougle, "but I can tell you that if you know autism, then you can see that the impaired social relatedness in those disorders is quite different - it's not autism."

Categorization of patients could be improved by the identification of biomarkers - molecular or physiological traits that correlate with specific symptoms or manifestations of ASD. Such markers could steer patient selection for clinical trials, as well

as illuminate autism's still-mysterious aetiology. Several candidate genes have been identified, although they have not yet been woven into a coherent model. In April 2012, a large-scale gene sequencing study of 343 families revealed more than 300 potential susceptibility factors - and an unexpectedly strong link to FXS, with many genes encoding targets regulated by fragile $\mathrm{X}$ mental retardation protein (FMRP $)^{4}$. "This will still need to be settled with empirical studies in humans," says Bear. In some cases, causative mutations may be exceedingly rare or even limited to certain bloodlines. For example, one recent genetic study identified a link between a defect in a metabolic gene and autism in two related families, and subsequent animal studies suggested that autistic individuals with this mutation might benefit from dietary supplementation ${ }^{5}$. This finding is unlikely to help the vast majority of people with autism, however, and the search continues for broader aspects of ASD pathology.

Some research-

"We have

become good at stealing drugs developed for other disorders." ers are also beginning to investigate the contributions of inflammatory immune responses and autoimmunity in causing, or at least exacerbating, autism. "Not long ago, talking about immune stuff was considered fringe and funky, and I'm not fringe or funky," says McDougle. "But I think that immune dysfunction is going to prove to be important in a very meaningful subgroup of patients - maybe as many as 10-25\%." While the evidence for this correlation remains circumstantial, other scientists are taking the possibility seriously. Stewart Johnson's maverick experiments with whipworm eggs were informed by research data suggesting that the pig parasitic worms, which do not permanently colonize the human gut, can suppress harmful inflammatory responses ${ }^{6}$. Johnson reported improvement in many of his son's behavioural symptoms, and his work was sufficiently persuasive to convince psychiatrist and autism specialist Eric Hollander at the Albert Einstein College of Medicine in New York to embark on a formal clinical trial of this treatment people with ASD .

Feedback from parents has led to other investigations of therapeutic modalities besides the whipworm. For example, input and financial support from parents led Hardan to conduct a clinical trial examining $\mathrm{N}$-acetylcysteine, an antioxidant and modulator of glutamate signalling, as a potentially milder alternative to antipsychotic drugs for irritability symptoms ${ }^{7}$. Fischbach notes that parents of children with autism helped call attention to a surprising observation that could offer novel insights into ASD pathology.
"One-quarter of the people in our study group of almost 3,000 families report that when their children have a mild fever, their social interaction improves - some start speaking or making more eye contact," he says. "We are trying to formulate controlled trials to understand that phenomenon better."

Not every anecdotal finding pays dividends - Veenstra-VanderWeele cites the prominent example of secretin, a digestive hormone that was described as an effective treatment for core deficits in autism based on a case report ${ }^{8}$ of three children published by a research group in 1998, but it ultimately led researchers down a costly rabbit hole. "We figured out three or four years later that it does absolutely nothing," says VeenstraVanderWeele. "People were spending a lot of time and effort pursuing that instead of something with more evidence behind it." The problem is that parents, desperate for treatment options, are confronted by a staggering amount of ambiguous data - and misinformation - and clinicians must take care to keep parents informed of what has been scientifically confirmed. Anagnostou says her top priority is to steer families pursuing alternative approaches away from known hazards, such as excessively high doses of vitamin B6 or omega-3 fatty acids, or unsafe treatments such as chelation therapy, which entails the use of compounds intended to remove heavy metals from the body. "I take all of the non-evidence-based things that they're interested in and tell them what's dangerous, and then I hope that they will stay in the non-dangerous territory," she says. "But the truth is, we haven't given them that many options."

With luck, this will change as the current round of clinical studies concludes. Clinicians and researchers eagerly await news of the first drug to successfully chip away at autism's core symptoms. However, VeenstraVanderWeele points out that even a runaway success on the pharmacological front will only be the starting point for effective therapy, enabling people with autism to receive the behavioural and educational training they need to function in society. "You can set the brain up to learn," he says, "but you still need to teach."

Michael Eisenstein is a freelance science writer based in Philadelphia, Pennsylvania.

1. Dölen, G. et al. Neuron 56, 955-962 (2007).

2. Michalon, A. et al. Neuron 74, 49-56 (2012).

3. Jacquemont, S. et al. Sci. Transl. Med. 3, 64ra1 (2011).

4. Iossifov, I. et al. Neuron 74, 284-299 (2012).

5. Novarino, G. et al. Science doi: 10.1126/ science.1224631 (2012).

6. Summers, R. W. et al. Am. J. Gastroenterol. 98, 2034-2041 (2003).

7. Hardan, A. Y. et al. Biol. Psychiatry 71, 956-961 (2012).

8. Horvath, K. et al. J. Assoc. Acad. Minor Phys. 9, 9-15 (1998). 